\title{
Desenvolvimento de Laboratório Remoto Utilizando Módulo Didático para Ensino de Microcontroladores
}

\author{
Flávio Henrique Toribio Destro ${ }^{1}$, Fábio Iaione ${ }^{2}$ \\ ${ }^{1,2}$ Faculdade de Computação - Universidade Federal de Mato Grosso do Sul (UFMS) \\ 79070-900 - Campo Grande - MS - Brasil \\ flavio_toribio@hotmail.com, iaione@facom.ufms.br
}

\begin{abstract}
Due to the growth of distance education in universities, laboratory activities are replaced by other more theoretical forms of teaching. However, in the area of microcontrollers, the laboratory in-person experience using hardware is much more interesting and useful for students. Given the above, this work proposes the development of a remote laboratory using a hardware module to teach microcontrollers. The student can interact through a Web browser with several functionalities of the hardware module, located remotely in a physical laboratory of the university. Thus, the student gains a real experience, as if he were in person in the laboratory.
\end{abstract}

Resumo. Devido ao crescimento do ensino a distância nas universidades, atividades de laboratório acabam sendo substituídas por outras formas mais teóricas de ensino. Porém, na área de microcontroladores, a experiência presencial no laboratório utilizando hardware é muito mais interessante e útil para os alunos. Dado o exposto, este trabalho propõe o desenvolvimento de um laboratório remoto usando um módulo de hardware para o ensino de microcontroladores. $O$ aluno pode interagir por meio de um navegador Web com várias funcionalidades do módulo de hardware, localizado remotamente em um laboratório físico da universidade. Sendo assim, o aluno ganha uma experiência real, como se estivesse presencialmente no laboratório.

\section{Introdução}

Com o recente crescimento da educação à distância nas universidades, é necessário desenvolver novas metodologias de ensino para atividades que geralmente são trabalhadas exclusivamente em laboratório. Tais atividades possuem componentes físicos que devem ser manipulados presencialmente, tornando-se um problema para um sistema de educação à distância. Por conta disso, na maioria das "universidades virtuais", os estudantes ainda precisam atender a eventos presenciais, tais como aulas de laboratório [Kaderali et al., 2001].

Uma das metodologias já apresentadas em diversos trabalhos [Djordjevic et al., 2005, Ko et al., 2001, Swamy et al., 2002, Tan et al., 2002] baseia-se inteiramente na simulação de placas de hardware via software. Isso soluciona o problema da presença física no laboratório, mas apresenta uma grande limitação, que é a incapacidade de um simulador reproduzir todas as limitações físicas e a complexidade de um sistema de hardware. Normalmente, a simulação não consegue considerar absolutamente todas as 
VIII Congresso Brasileiro de Informática na Educação (CBIE 2019)

Anais do XXX Simpósio Brasileiro de Informática na Educação (SBIE 2019)

variáveis que influenciam o sistema de hardware. Alguns estudos concluíram que o desenvolvimento das habilidades "práticas" do aluno, por meio da execução de experimentos reais envolvendo sistemas embarcados, por exemplo, são essenciais para uma boa aprendizagem nessa área de estudo [Cooper, 2000]. Portanto, desenvolver metodologias de ensino a distância que apresentem uma experiência mais "hands-on" com laboratórios virtuais, continua sendo um desafio [Eppes and Schuyler, 2004]. Devido a fatores desfavoráveis como custo, espaço e tempo, referentes ao ensino em laboratório presencial [Luthon et al., 2009], soluções alternativas para a introdução de laboratórios remotos mais "reais" continuam sendo um assunto discutido na literatura.

Existem vários trabalhos já desenvolvidos com o objetivo de solucionar o problema dos experimentos práticos no ensino a distância, na área de hardware. Dentre eles, pode-se citar o DIESEL, o REDLART e o LaboRem.

O DIESEL (Distant-Internet Embedded Systems Engineering Laboratory) [Yue et al., 2009] foi um projeto desenvolvido na University of Ulster, que oferece uma solução modular para o problema. O sistema também é genérico no sentido de aceitar vários tipos de sistemas embarcados como microcontroladores, DSPs, FPGAs e SoCs em sua plataforma. Os autores utilizaram um design escalável, que aceita adições futuras de novos tipos de sistemas embarcados. A parte de experimentação do sistema possui a compilação remota de firmware, e a visualização remota dos instrumentos utilizados (osciloscópio, gerador de função, multímetro e analisador lógico) pela interface do cliente, que é providenciada pelo sistema LabVIEW, da National Instruments. Foram utilizados dois módulos de hardware para a comunicação e interação entre as placas de experimento e o computador, que age como servidor no laboratório, o EBIM (Experiment Board Interface Module) e o IIM (Instrumentation Interface Module). Esses módulos que possuem a função de "middleware" entre o servidor e os circuitos constituem um sistema extensivo e complexo, formado por vários componentes com funções diferentes.

O REDLART (REconfigurable Digital Laboratory for Advanced Research and Teaching) [Moreira, 2009] tem o objetivo de trazer a prototipação rápida de novos experimentos em sistemas digitais nas áreas de engenharia, telecomunicações, computação e afins, através de dispositivos semicondutores do tipo FPGA. O sistema desenvolvido também é modular, permitindo que o foco dos experimentos seja maior no problema que se deseja resolver, e não na complexidade do hardware utilizado, que é isolada pelo conjunto de serviços de software oferecido pelo sistema.

O LaboRem [Luthon et al., 2009] é uma arquitetura de instrumentação remota de um laboratório virtual para estudantes de graduação cursando matérias que abordam processamento de sinais, depuração de circuitos e testes de sistemas. Esse sistema tem uma abordagem mais voltada aos jogos educacionais, tornando o ensino mais didático. O sistema também é implementado com LabVIEW, mas possui o adicional do streaming de vídeo em tempo real do circuito.

Dado o exposto, este trabalho tem como objetivo desenvolver um laboratório remoto, baseado em um módulo didático de hardware, para o ensino de microcontroladores. O aluno poderá interagir via interface World Wide Web (WWW) com várias funcionalidades do módulo didático, localizado remotamente em um 
VIII Congresso Brasileiro de Informática na Educação (CBIE 2019)

Anais do XXX Simpósio Brasileiro de Informática na Educação (SBIE 2019)

laboratório físico da universidade, e disponível em qualquer dia e horário. Tais interações podem ser analisadas pelo streaming de vídeo que é transmitido em tempo real durante o experimento, mostrando os visores do módulo didático. Como a solução apresentada não é baseada em simulação, os alunos terão uma experiência real, como se estivessem presentes no laboratório, melhorando a aprendizagem.

\section{Metodologia}

Nessa seção são descritos os materiais e métodos utilizados no desenvolvimento do laboratório remoto, envolvendo hardware, software e firmware.

\subsection{Módulo Didático para Ensino de Microcontroladores}

O módulo principal utilizado no sistema corresponde a placa de hardware BIG8051 (Figura 1). O módulo didático BIG8051, fabricado pela MikroElektronika, é um sistema que fornece um ambiente de desenvolvimento para a programação e experimentação de microcontroladores com núcleo CIP-8051 (núcleo MCS-51 acrescido de pipeline), da Silicon Laboratories. A placa é fornecida com o microcontrolador C8051F040, que executa até $25 \mathrm{MIPS}$ (@25 MHz), e possui internamente: $64 \mathrm{kB}$ de memória flash (programa), 4352 bytes ( 256 bytes do núcleo MCS-51 acrescido de $4 \mathrm{kB}$ ) de memória RAM (dados), 64 pinos de I/O, portas seriais UART (duas), SPI, I2C, CAN, cinco temporizadores/contadores (16 bits) de uso geral, matriz programável de temporizadores/contadores (16 bits) com seis módulos de captura/comparação, conversor analógico-digital (ADC) e digital-analógico (DAC) de 12 bits, tensão de referência, três comparadores de tensão, entre outros. A placa pode ser programada e depurada utilizando-se um módulo USB, conectado via interface JTAG ao microcontrolador.

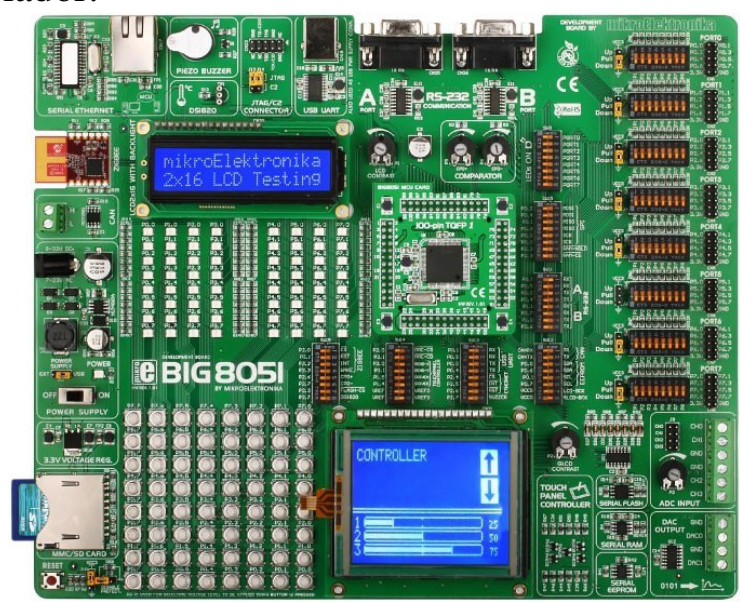

Figura 1. Módulo didático BIG8051 para ensino de microcontroladores $(26,5 \times 22 \mathrm{~cm})$.

No módulo BIG8051, externamente ao microcontrolador, estão disponíveis os seguintes dispositivos: 64 teclas e 64 LEDs, para definição e visualização dos sinais nos 64 pinos de I/O; conectores para acesso direto aos 64 pinos de I/O do microcontrolador; buzzer piezoelétrico; display de cristal líquido alfanumérico (2 linhas x 16 caracteres); display de cristal líquido gráfico (128 x 64 pixels); memória EEPROM I2C de 128 bytes; memória SRAM SPI de 8 kB; memória flash SPI de 8 Mbits; conector para 
VIII Congresso Brasileiro de Informática na Educação (CBIE 2019)

Anais do XXX Simpósio Brasileiro de Informática na Educação (SBIE 2019)

cartões de memória MMC/SD; conectores ethernet RJ-45, EIA/TIA-232 DB-9 (2), USB (fonte de energia e porta serial virtual); conectores para 4 entradas e 2 saídas analógicas; conector para sensor de temperatura digital; e conector para módulo de RF ZigBee, entre outros. Como a quantidade de pinos de $\mathrm{I} / \mathrm{O}$ do microcontrolador é insuficiente para a conexão simultânea de todos os dispositivos presentes na placa, existem chaves (DIP switches) que permitem a conexão de um ou outro dispositivo aos pinos de I/O [MikroElektronika, 2010].

A programação do microcontrolador pode ser feita em linguagem de montagem ou em linguagens de programação de alto nível, sendo a linguagem $\mathrm{C}$ a mais utilizada.

\subsection{Arquitetura do Sistema}

O sistema desenvolvido consiste de um lado servidor e um lado cliente. O lado cliente é a interface web utilizada pelo aluno, que acessa remotamente o laboratório de ensino utilizando um aplicativo "navegador". Já o lado servidor é responsável pela hospedagem do website, pela hospedagem da webcam, e pela interface entre o cliente do laboratório remoto e a placa BIG8051, utilizando um módulo programador, e um Arduino Mega 2560 para gerar e ler alguns sinais (Figura 2).

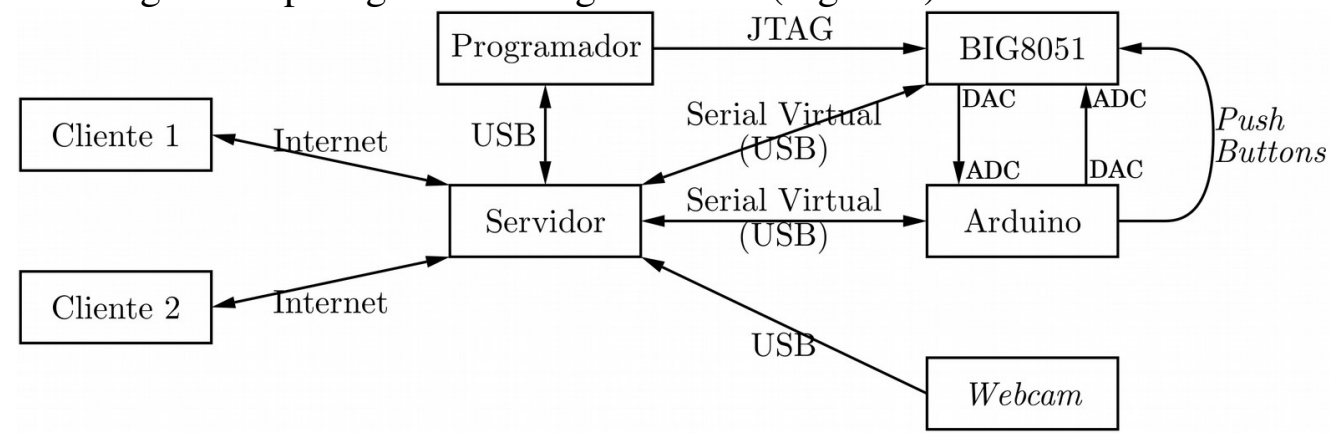

Figura 2. Diagrama de blocos do sistema.

\subsection{Lado Cliente}

O lado cliente é uma página web (Figura 3) constituída de seis componentes: push buttons, controle de tensão para enviar ao ADC, monitor de tensão lida na saída do DAC, webcam, console serial e editor de código (compilador/uploader).

Todas as requisições feitas pelo cliente (ex.: um clique em um push button ou no botão "Compilar") são enviadas para o servidor através do protocolo WebSocket [Lombardi, 2015], resultando em uma maior velocidade de comunicação entre os pares devido à sua conexão persistente. Isso é altamente desejável em uma aplicação de tempo-real como a apresentada aqui, sendo a única desvantagem o fato dessa tecnologia estar disponível somente em navegadores mais atuais que suportam HTML5.

A página envia uma requisição ao servidor tanto ao pressionar, como ao soltar os push buttons da Figura 3, simulando não apenas o clique como também o click and hold de uma aplicação real. O middleware - que simula o clique do botão - adiciona um bounce [Morton, 2001] de 500 microssegundos a cada mudança de estado do componente. Isto faz com que o sistema comporte-se como uma tecla real, forçando o aluno a utilizar um algoritmo de debouncing no firmware do microcontrolador. 
VIII Congresso Brasileiro de Informática na Educação (CBIE 2019)

Anais do XXX Simpósio Brasileiro de Informática na Educação (SBIE 2019)

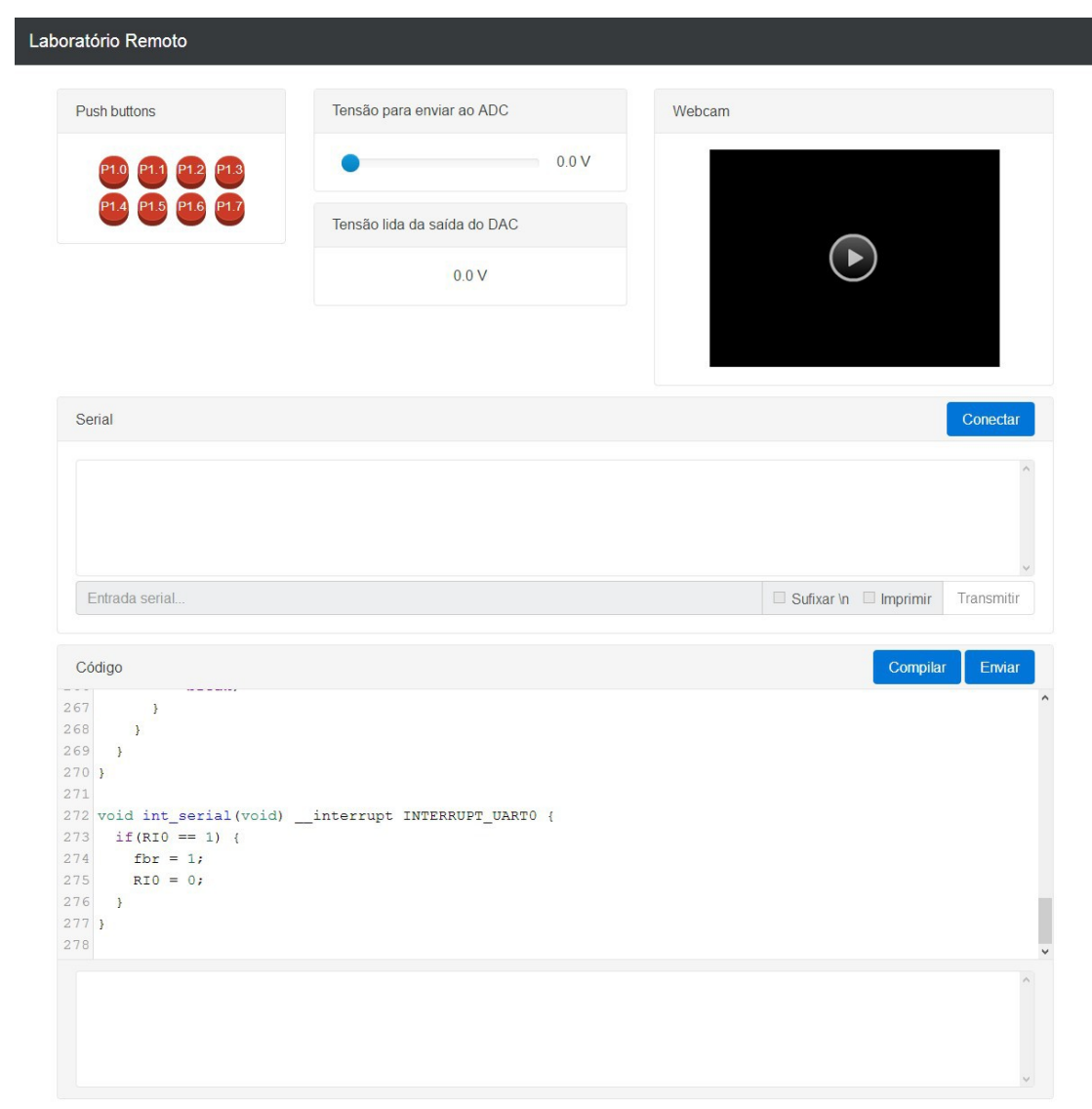

Figura 3. Visão geral do front-end do website.

Por meio de um componente slider, o usuário pode enviar uma tensão elétrica entre 0 e 3,3 Volts para o conversor analógico-digital do microcontrolador (canal 0).

A tensão elétrica gerada pelo conversor digital-analógico (DAC) do microcontrolador pode ser visualizada no componente da Figura 3, que é atualizado a cada $250 \mathrm{~ms}$ (apenas se houver mudança, para economizar banda de rede).

A webcam (Figura 3) pode ser utilizada para visualizar certos dispositivos da placa BIG8051, como por exemplo, os textos e imagens mostrados pelos dois displays de cristal líquido e pela matriz de LEDs. A resolução de vídeo e a taxa de atualização de quadros da webcam são dependentes do servidor específico hospedando a aplicação. Nos testes de atraso de quadros, foi configurada uma taxa de quadros de 20 FPS e resolução de 320x240 pixels.

Por meio do Console serial, o usuário pode interagir com a porta serial UART do microcontrolador da BIG8051. Todos os caracteres enviados pelo microcontrolador são mostrados nesse console, assim como, caracteres digitados no console podem ser enviados ao microcontrolador.

$\mathrm{O}$ usuário deve escrever o firmware do microcontrolador em linguagem $\mathrm{C}$, no componente Código (Figura 3), podendo compilá-lo e depois enviá-lo à placa BIG8051. 
VIII Congresso Brasileiro de Informática na Educação (CBIE 2019)

Anais do XXX Simpósio Brasileiro de Informática na Educação (SBIE 2019)

\subsection{Lado Servidor}

O lado servidor é implementado em um computador localizado na instituição de ensino, possuindo os seguintes objetivos:

- Hospedar um servidor HTTP e WebSocket;

- Compilar e gravar os firmwares na placa didática BIG8051;

- Interagir com a placa BIG8051 por meio de um Arduino Mega 2560 e de uma porta serial UART virtual (USB);

- Hospedar um servidor RTMP para transmitir, em tempo real, imagens capturadas da placa didática pela webcam.

O servidor HTTP e WebSocket foi programado usando os frameworks Express e Socket.IO do Node.js. Esta solução acabou sendo elegante devido à natureza assíncrona do protocolo WebSocket. Anteriormente na etapa de levantamento de requisitos e projeto da aplicação, haviam sido pensadas outras alternativas que necessitariam de codificações mais complexas para implementar a comunicação entre o servidor HTTP e WebSocket. Por exemplo, PHP com Apache e Ratchet, Ruby on Rails com Faye WebSockets, entre outros. A codificação é quase que totalmente feita em JavaScript, exceto nos casos de arquivos de configurações, arquivos de estilo em cascata e/ou arquivos de views.

O compilador utilizado é o Small Device C Compiler (SDCC), sendo que a compilação é realizada através da criação de um processo do SDCC, diretamente pelo back-end do servidor. Quando o usuário envia a ação de compilar, a aplicação envia uma requisição WebSocket com o código do usuário, e faz o pré-processamento para checar se há alguma violação de segurança (como por exemplo a inclusão de arquivos de cabeçalho com caminho de diretórios do sistema). Após essa etapa, é iniciado o processo de compilação e acoplamento. Em qualquer uma dessas etapas, se houver um erro, o usuário recebe uma mensagem imediatamente. A gravação do firmware é realizada utilizando o módulo USB Debug Adapter/Programmer, da Silicon Laboratories, ligado diretamente no conector JTAG da placa didática, e a mesma rotina de criação do processo de compilação, porém, o processo criado é o da ferramenta FlashUtilCL.exe. Esta ferramenta está disponível apenas para Microsoft Windows, sendo esse o motivo do sistema desenvolvido funcionar apenas neste sistema operacional. No entanto, o sistema já está preparado para outras plataformas, como Linux e Mac OS, considerando a existência da ferramenta de gravação para esses sistemas operacionais.

Como mostrado na Figura 2, o Arduino funciona como um middleware, simulando o pressionamento de 8 teclas (push buttons) da placa BIG8051, colocando pinos de I/O do microcontrolador em nível lógico 0 exatamente da mesma forma que as teclas fazem ao serem pressionadas. Os pinos P2 a P9 do Arduino controlam os níveis lógicos aplicados aos pinos P1_0 a P1_7, includindo bounce de 500 microssegundos ao pressionar e ao soltar. Além disso, o Arduino lê tensões elétricas geradas pelo conversor digital-analógico (DAC), por meio da conexão entre a saída DAC0 da BIG8051 e a entrada A0 do Arduino. Ainda, o Arduino envia tensões elétricas para o conversor analógico-digital (ADC) do módulo BIG8051, por meio de seu pino P11 (configurado 
VIII Congresso Brasileiro de Informática na Educação (CBIE 2019)

Anais do XXX Simpósio Brasileiro de Informática na Educação (SBIE 2019)

como saída PWM-Pulse Width Modulation), que para fornecer um sinal realmente analógico passa por um filtro passa-baixas $\mathrm{RC}(\mathrm{Fc}=10 \mathrm{~Hz})$, antes de ser aplicado ao pino CH0 (canal 0 do ADC) da BIG8051. A comunicação entre o Servidor e o Arduino é realizada por meio de uma porta serial UART virtual (115200 bps), via USB, e o protocolo usado é extremamente simples, composto de um byte "ação a tomar" e um byte de parâmetro da ação.

A comunicação serial UART entre o servidor e a placa didática BIG8051 é realizada por meio de uma porta serial virtual, implementada via porta USB. Esta ligação é utilizada para estabelecer a comunicação serial UART entre o cliente e o microcontrolador (9600 bps, 8N1). A comunicação serial com a placa BIG8051, como também com o Arduino, é realizada através da biblioteca Node Serialport1, do Node Package Manager (NPM). O administrador do servidor pode configurar as portas COM por busca automática ou especificá-las manualmente no arquivo de configurações app/config/index.js.

O conjunto de servidor e cliente RTMP (Real Time Messaging Protocol) é responsável pelo broadcast da webcam para os usuários. Em etapas anteriores do desenvolvimento do trabalho, foi especificado o uso de um formato de streaming de vídeo capaz de ser visualizado diretamente em navegadores que suportam HTML5. Foram levantados os requisitos dos formatos HLS, Dash e MJPEG. A escolha final foi o formato RTMP, devido aos seguintes benefícios: streaming em tempo real, bom suporte e fácil implementação. O servidor RTMP utilizado foi o Nginx RTMP Module, que é fácil de configurar e mostra-se extremamente escalável. Para a configuração final do servidor, foram necessárias apenas 17 linhas.

No caso do cliente RTMP, pode-se utilizar qualquer software. Durante o desenvolvimento do trabalho, o software que se apresentou mais confiável foi o Adobe Flash Media Live Encoder. Outras alternativas são: Open Broadcaster Software (OBS), ffmpeg, e outros.

Cabe observar que o servidor atende um cliente por vez, pois existe apenas um módulo didático conectado ao mesmo. Quando um segundo cliente tenta utilizar o sistema simultaneamente, este recebe uma mensagem de ocupado no navegador. $\mathrm{O}$ sistema é liberado quando o cliente que está usando o sistema fecha a aba do seu navegador, ou fica inativo por três minutos.

\section{Testes e Resultados}

Nesse capítulo são apresentados os testes realizados e os resultados obtidos, após a implementação do sistema. Os testes contemplam a verificação do correto funcionamento do sistema, assim como os atrasos de tempo na interação entre clienteservidor, considerando duas situações, cliente-servidor na mesma intranet e clienteservidor na internet. Os atrasos na internet foram verificados entre dois pares localizados em diferentes estados do país, Mato Grosso do Sul e Paraná.

\subsection{Atraso da Webcam}

$\mathrm{Na}$ intranet, o método de medição do atraso utilizou uma câmera para capturar a tela do cliente em conjunto com a tela do próprio servidor, na qual era visualizado um 
VIII Congresso Brasileiro de Informática na Educação (CBIE 2019)

Anais do XXX Simpósio Brasileiro de Informática na Educação (SBIE 2019)

cronômetro com precisão de milissegundos (Figura 4). Ao fotografar 40 quadros arbitrários do cenário, pode-se verificar o atraso de tempo para uma imagem capturada pela webcam no servidor, aparecer na tela do cliente. A média de atraso da webcam na intranet foi de $676,87 \mathrm{~ms}$, com desvio padrão de $110,94 \mathrm{~ms}$.

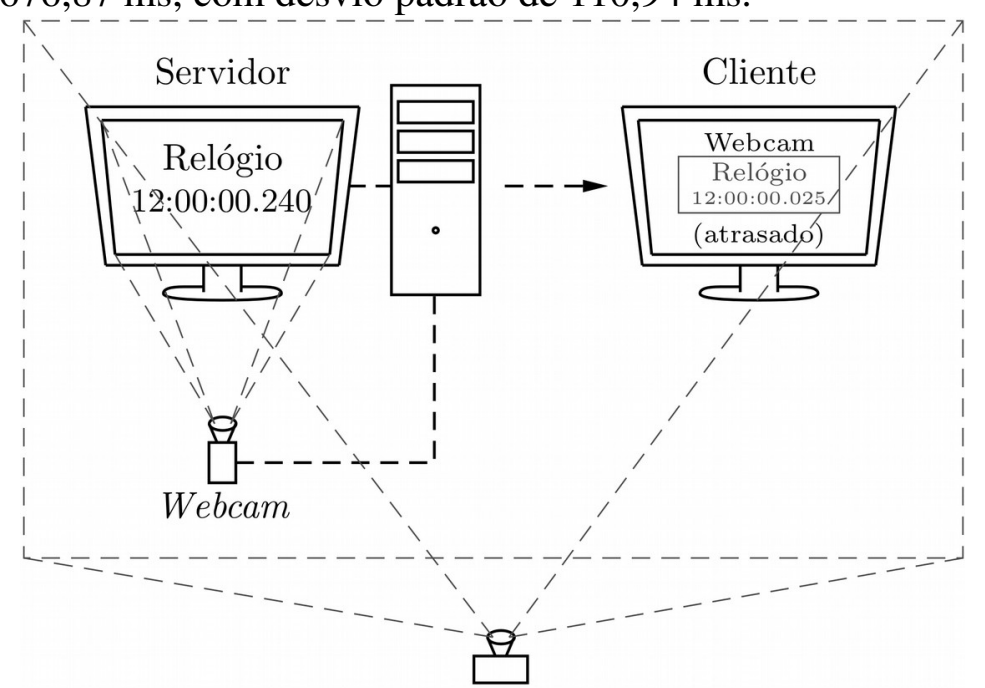

Figura 4. A webcam está filmando um cronômetro no próprio servidor, que então envia o vídeo para o cliente, que é visualizado no navegador. Percebe-se que nesse exemplo houve um atraso de 12:00:00.240-12:00:00.025 $=215 \mathrm{~ms}$.

O atraso da webcam na internet foi verificado baseando-se na sincronização via NTP [Mills, 1991], entre o relógio do servidor e do cliente. A sincronização foi feita com precisão de segundos entre dois pares em diferentes estados do país. Novamente, para medir a diferença dos tempos, a webcam foi posicionada de forma a filmar o relógio do servidor, para então o cliente capturar a tela de visualização da webcam e seu próprio relógio, sincronizado anteriormente com o servidor. A média de atraso da webcam na internet foi de $1 \mathrm{~s}$, com desvio padrão de $0,5 \mathrm{~s}$.

\subsection{Atraso da Comunicação Serial}

O método utilizado no atraso da comunicação serial baseou-se na medição do tempo de ida e volta de um caractere transmitido do cliente para o servidor, executando no módulo BIG8051 um firmware que implementava um "ping-pong" na porta serial (transmitia para o cliente um caractere, imediatamente após receber um caractere).

$\mathrm{Na}$ intranet, a média do atraso de ida e volta de um byte foi de $21,82 \mathrm{~ms}$, com desvio padrão de $7,68 \mathrm{~ms}$. Na internet, a média de atraso de ida e volta foi de $132,97 \mathrm{~ms}$, com desvio padrão de 22,23 ms.

\subsection{Atraso dos Push Buttons}

A verificação do atraso dos push buttons foi realizada pela medição do tempo entre o clique inicial do usuário e o retorno de um byte pela porta serial (console serial). Foram coletadas 40 amostras de tempo de atraso. A média de atraso na intranet foi de $15,32 \mathrm{~ms}$, com desvio padrão de $4,26 \mathrm{~ms}$, e na internet foi $29,05 \mathrm{~ms}$, com desvio padrão de $9,44 \mathrm{~ms}$. 
VIII Congresso Brasileiro de Informática na Educação (CBIE 2019)

Anais do XXX Simpósio Brasileiro de Informática na Educação (SBIE 2019)

\subsection{Atraso do DAC e ADC}

A verificação de atraso dos componentes DAC e ADC foi realizada simultaneamente. Um valor de tensão foi transmitido pelo slider de tensão do componente ADC (Figura 3), e um firmware de teste na BIG8051 mediu essa tensão e gerou o mesmo valor no seu DAC, que por sua vez, atualizou o componente DAC da página (Figura 3). Na intranet, a média do atraso foi de 73,70 ms, com desvio padrão de $6,96 \mathrm{~ms}$, e na internet foi de $235,35 \mathrm{~ms}$, com desvio padrão de 11,01 ms.

\section{Conclusão}

O laboratório remoto desenvolvido fornece ao aluno, participante de uma turma à distância, a possibilidade de utilizar um módulo didático real, para o ensino de microcontroladores. O sistema proporciona uma interação entre o aluno e a placa didática, à distância, que é como se o aluno estivesse presencialmente no laboratório. Com o uso de streaming de vídeo, o aluno pode visualizar os dois displays de cristal líquido e a matriz de LEDs da placa. Além disso, pode realizar o pressionamento de push buttons (com bouncing), gerar tensões para serem aplicadas na entrada do ADC, ver a tensão gerada na saída do DAC, e interagir com a porta serial do microcontrolador por meio de um console serial. O sistema proporciona uma forma de escrever o firmware para o microcontrolador da placa didática em linguagem $\mathrm{C}$, utilizando apenas o navegador, sem a instalação de IDEs, compiladores e outras ferramentas. Cabe observar que utilizando o sistema proposto é possível realizar uma grande variedade de experimentos, pois além dos recursos internos do microcontrolador, pode-se utilizar as memórias EEPROM (I2C) e flash (SPI) disponíveis na placa, assim como outros dispositivos.

Levando em consideração os resultados obtidos nos testes do sistema, é aceitável afirmar que a aplicação atende às propostas iniciais de forma satisfatória. Um dos desafios analisados no começo do projeto foi o atraso existente na interação do aluno com o laboratório, devido a todos os obstáculos que um pacote de dados percorre desde o momento que é enviado pelo servidor, até o momento que é recebido e visualizado na tela do cliente. Mesmo assim, o sistema apresentou um atraso perfeitamente aceitável para o vídeo $(1 \pm 0,5 \mathrm{~s})$. Os outros componentes também apresentaram atrasos relativamente pequenos: 132,97 ms para a comunicação serial, 129,05 ms para os push buttons e 235,35 ms para o DAC/ADC. Portanto, pode-se afirmar que o sistema alcançou seu objetivo inicial, superando um dos maiores desafios analisados no levantamento de requisitos.

Inicialmente, o sistema desenvolvido será utilizado na disciplina que aborda microcontroladores, no curso de Engenharia de Computação, permitindo a realização de experimentos extraclasse. Assim, a carga horária da disciplina que pode ser ministrada à distância $(10 \mathrm{~h})$ poderá incluir aulas de laboratório. Além disso, o sistema permitirá a reexecução de experimentos realizados presencialmente, melhorando o entendimento e a fixação dos conceitos. Em um segundo momento, pretende-se utilizar o feedback dos alunos para implementar melhorias no sistema.

Dado o exposto, acredita-se que esse trabalho contribuiu com a área de ensino a distância, especificamente na área de laboratórios remotos. 
VIII Congresso Brasileiro de Informática na Educação (CBIE 2019)

Anais do XXX Simpósio Brasileiro de Informática na Educação (SBIE 2019)

\section{References}

Cooper, M. (2000). The challenge of practical work in an euniversity - real, virtual and remote experiments. In Information Society Technologies Conference: The Information Society for All, Proceedings of the, páginas 34-40.

Djordjevic, J., Nikolic, B., e Milenkovic, A. (2005). Flexible webbased educational system for teaching computer architecture and organization. Education, IEEE Transactions on, 48(2):264-273.

Eppes, T. e Schuyler, P. (2004). Work in progress - a distance laboratory system using agilent test equipment. In Frontiers in Education, 2004. FIE 2004. 34th Annual, páginas T3C/20-T3C/21 Vol. 1.

Kaderali, F., Steinkamp, G., e Cubaleska, B. (2001). Studying electrical engineering in the virtual university. Internal Journal on Engineering Education, páginas 119-130.

Ko, C. C., Chen, B., Hu, S., Ramakrishnan, V., Cheng, C. D., Zhuang, Y., e Chen, J. (2001). A web-based virtual laboratory on a frequency modulation experiment. Systems, Man, and Cybernetics, Part C: Applications and Reviews, IEEE Transactions on, 31(3):295-303.

Lombardi, A. (2015). WebSocket: Lightweight Client-Server Communications, página 1. O'Reilly Media.

Luthon, F., Petre, A., Steriu, D., e Besleaga, A. (2009). Laborem: open lab for remote work. In Signals, Circuits and Systems (SCS), 2009 3rd International Conference on, páginas $1-6$.

MikroElektronika (2010). BIG8051 Development System User Manual. MikroElektronika. Disponível em: http://www.mikroe.com/big8051/ [visitado em 02/03/2015].

Mills, D. L. (1991). Internet time synchronization: the network time protocol. IEEE Transactions on Communications, 39(10):1482-1493.

Moreira, V. R. (2009). Plataforma em hardware reconfigurável para o ensino e pesquisa em laboratório de sistemas digitais a distância. Universidade Estadual de Campinas (UNICAMP). Faculdade de Engenharia Elétrica e de Computação. Dissertação de mestrado. Orientador Arantes, D. S.

Morton, J. (2001). Exploring the pic5x series. In PIC: Your Personal Introductory Course, IDC Technology, páginas 59-60. Newnes, $2^{\text {a }}$ edição.

Swamy, N., Kuljaca, O., e Lewis, F. (2002). Internet-based educational control systems lab using netmeeting. Education, IEEE Transactions on, 45(2):145-151.

Tan, K. K., Lee, T. H., e Soh, C. Y. (2002). Internet-based monitoring of distributed control systems-an undergraduate experiment. Education, IEEE Transactions on, 45(2):128-134.

Yue, X., Drakakis, E. M., Harkin, J., Callaghan, M. J., McGinnity, T. M, Maguire, L. P. (2009). Modular hardware design for distant-internet embedded systems engineering laboratory. Computer Applications in Engineering Education, 17(4):389-397. 